\title{
Advances in Tuberculosis: Diagnostics
}

\author{
Varinder Singh ${ }^{1} \cdot$ Sushil K. Kabra ${ }^{2}$
}

Received: 19 March 2019 / Accepted: 20 March 2019 / Published online: 3 April 2019

(C) Dr. K C Chaudhuri Foundation 2019

Tuberculosis in children is a well identified morbidity. In 2017, an estimated 10.0 million people (range, 9.0-11.1 million) developed TB disease globally. This includes 5.8 million men, 3.2 million women and 1.0 million children. Precise magnitude of pediatric tuberculosis is not known but it is estimated that about $10 \%$ of total burden of tuberculosis is among children. In India, estimated 2.2 lakh children become ill with tuberculosis (TB) each year (22\% of global TB burden), with a slightly higher burden among males. Pulmonary TB is the most common form in children but the extrapulmonary TB forms a larger proportion of cases than in adults [1]. The advances in the TB diagnostics and treatment are being enlarged to cover all age populations, including children.

The Indian Journal of Pediatrics, is bringing out a special symposium on advances in pediatric tuberculosis. Articles written by experts on various aspects of childhood tuberculosis shall be included in this two part symposium. The current issue of the journal includes articles related to the diagnostics of tuberculosis in children.

Diagnosis of childhood tuberculosis is a challenge due to the pauci-bacillary nature of infection and the difficulty in obtaining appropriate sample. The first article on advances in the diagnosis by Wattal et al. discusses details of new tests introduced over a past few decades for the diagnosis of tuberculosis (TB) with special focus on those which have been evaluated for their application in pediatric tuberculosis [2]. There is an attempt to improve smear microscopy by introducing light-emitting diode (LED) fluorescence microscopy and there are also some automated digital microscopy platforms under evaluation. Introduction of automated liquid culture

Sushil K. Kabra

skkabra@hotmail.com

1 Department of Pediatrics, Kalawati Saran Children's Hospital and Lady Hardinge Medical College, New Delhi, India

2 Department of Pediatrics, All India Institute of Medical Sciences, New Delhi 110029, India platform along with rapid molecular based identification methods have considerably reduced the time delay in mycobacterial culture. Recent addition of many nucleic acid amplification platforms like Amplicor PCR ${ }^{\mathrm{TM}}$, Genprobe ${ }^{\mathrm{TM}}$, Xpert MTB/Rif ${ }^{\mathrm{TM}}$, line probe assays like Hain's Test, loop mediated isothermal amplification, etc. have widened the choice of tests available for the bacteriological diagnosis of TB and also the rapid detection of resistance to anti-TB drugs. Latest techniques like microarray and gene sequencing are also being used in clinical laboratories with variable results. Tuberculin skin test and $\mathrm{T}$ cell based interferon-gamma release assays have their role primarily in the diagnosis of latent TB infection or as an indirect evidence for likely TB disease. Biomarkers are the latest addition in the battery of TB diagnostic tests facilitating diagnosis using easily accessible samples like urine, blood and breath of patients. Many biomarkers are still under evaluation and some of them are found to have a potential role as promising diagnostic tests of future.

Imaging is an integral part in the diagnosis of pulmonary tuberculosis in children. The article by Naranje et al. discusses the role of various imaging modalities like chest radiography, ultrasonography, computed tomography, magnetic resonance imaging, and functional scans like FDG PET CT [3]. Authors have detailed about the optimal use X-ray film of chest and its interpretations. They also discuss indications for CT scan, its interpretation and utility for monitoring the response. The article also addresses the issue of utility of MRI and functional PET CT scan in pediatric pulmonary tuberculosis. Chest Ultrasonography (USG) is an emerging investigation and its role in the diagnosis and monitoring is evolving. Naranje et al. discuss current status of USG specifically in the context of pulmonary tuberculosis [3].

TB can affect almost any organ system of the body. The clinical manifestations of extra-pulmonary tuberculosis (EPTB) are often non-specific. Imaging findings are also not always very specific thus making the diagnosis of EPTB more challenging than pulmonary tuberculosis. The article by Dawani et al. describes the imaging spectrum and role of imaging modalities such as USG, CT Scan and MRI in different types of extra-pulmonary tuberculosis [4]. 
Apart from advances in diagnosis, significant changes have occurred in the dosages of antituberculosis drugs in treatment of pediatric tuberculosis. The article by Mukherjee et al. provides the review of various studies on pharmacokinetics of first-line antituberculosis drugs [5]. It transcends the journey of change in the dosages for children with TB over past few decades. Determining the optimal dosages of isoniazid, rifampicin, pyrazinamide and ethambutol in children is necessary to obtain therapeutic serum concentrations of these drugs. Revised dosages have improved the exposure of 1st line anti-tubercular drugs to some extent; there is still a scope for modification of the dosages to achieve exposures which can lead to favourable outcome of the disease. High dose of rifampicin is being investigated in clinical trials in adults with some benefit and similar studies are required in children. Inter-individual pharmacokinetic variability and the effect of age, nutritional status, human immunodeficiency virus (HIV) infection and acetylator genotype may need to be accounted for in striving for the dosages best suited for an individual.

\section{Compliance with Ethical Standards}

Conflict of Interest None.

\section{References}

1. Global Tuberculosis Report 2018. Geneva: World Health Organization; 2018. Licence: CC BY-NC-SA 3.0 IGO.

2. Wattal C, Raveendran R. Newer diagnostic tests and their application in pediatric TB. Indian J Pediatr. 2019. https://doi.org/10.1007/ s12098-018-2811-0.

3. Naranje P, Seith Bhalla A, Sherwani P. Chest tuberculosis in children. Indian J Pediatr. 2019. https://doi.org/10.1007/s12098-018-02847-7.

4. Dawani A, Gupta AK, Jana M. Imaging in pediatric extra-pulmonary tuberculosis. Indian J Pediatr. 2019. https://doi.org/10.1007/s12098019-02858-y.

5. Mukherjee A, Lodha R, Kabra SK. Pharmacokinetics of first-line anti-tubercular drugs. Indian J Pediatr. 2019. https://doi.org/10. 1007/s12098-019-02911-w.

Publisher's Note Springer Nature remains neutral with regard to jurisdictional claims in published maps and institutional affiliations. 